\title{
The Knowledge Map of Intelligent System
}

\author{
Chunxue Wang ${ }^{1, a}$, Xiao Suo ${ }^{2, b}$ and Shuran Lv' \\ ${ }^{1}$ School of Safety and Environmental Engineering, Capital University of Economics and Business, \\ Beijing100070, China; \\ ${ }^{2}$ College of Resources and Safety Engineering, China University of Mining and Technology( Beijing), \\ Beijing100083, China. \\ alittlemonkey0325@126.com, bbruce88163@126.com, 'Isr22088@126.com
}

Keywords: intelligent system, knowledge map, information visualization, Citespace

\begin{abstract}
With the aim of drawing the knowledge map and understanding the research hotspot on intelligent system, the articles published from 2010 to 2015 have been searched with the keyword "intelligent system" based on the Web of Science database. The knowledge map of intelligent system was drawn by utilizing the CiteSpace software. Active countries / regions, institutions and research hotspots of intelligent system have been reached. The result shows that the United States, Europe and Asia are the most active areas of intelligent system studies; Islamic Azad University is on the top of research agencies in number of articles. From the perspective of the key words, power-systems, sliding-mode control, waves, supervisory control and traffic flow have the highest centrality; from the point of article co-citation analysis, the humanoid intelligent robot, the traffic flow management, the vibration are the hotspots of research in the field of intelligent system.
\end{abstract}

\section{Introduction}

In recent years, the intelligent system has been extensively studied all over the word. Sorting the hotspot of intelligent system is particularly necessary as it has always been the focus of attention and has a significant impact on human society.

The scientific knowledge map is a new research method which reveals the development process and the structural relationship of knowledge through data mining, information processing, and graphics rendering [1]. It can find out the knowledge base and discovery the research hotspot by the visualization analysis of scientific knowledge, which has good instruction significance to scientific research. The paper will take the intelligent system as a keyword and use the scientific knowledge map to analysis it.

\section{Data sources and methods}

The data of this paper are obtained from the Web of Science database, and 9706 records are retrieved. These following conditions are retrieved: using the Web of Sciencetm Core Collection as a database, taking the intelligent system as a keyword and the time span is from 2010 to 2015. 24059 authors, 1776 journals, 262636 citations and 10257 keywords are counted by the Histcite software. The data are visual analyzed through the CiteSpace III software after preliminary analysis.

\section{The knowledge distribution of intelligent system}

\subsection{The articles distribution of countries/regions and institutions.}

The geographical distribution heat map of scientific research institutions is drawn by using the Google Fusion Tables [2], the North America, Europe and Asia are the active areas of intelligent system research as is shown in Fig.1. The top 5 countries and institutions of issued amount are obtained by utilizing the Histcite software, the rank of countries / institutions of issued amount are shown in Table 1 and Table 2: The first three countries are China (1841), the United States (1529), 
Spain (671), while the first three institutions are Islamic Azad University (172), Chinese Academy of Sciences (141) and Tsinghua University (81).

Table 1 Rank of countries of issued amount

\begin{tabular}{ccc}
\hline Rank & Country & Amount \\
\hline 1 & China & 1841 \\
2 & The United States & 1529 \\
3 & Spain & 671 \\
4 & Iran & 620 \\
5 & South Korea & 514 \\
\hline
\end{tabular}

Table 2 Rank of institutions of issued amount

\begin{tabular}{ccc}
\hline Rank & Institution & Amount \\
\hline 1 & Islamic Azad University & 172 \\
2 & Chinese Academy of Sciences & 141 \\
3 & Tsinghua University & 81 \\
4 & University of Tehran & 76 \\
5 & Hong Kong Polytech Univ & 67 \\
\hline
\end{tabular}

\subsection{Research hotspot in the field of intelligent system.}

Each article that indexed by the Web of Science database will be subject categorized and it contains keyword labels. The research hotspot can be dug out under the detection of nodes which increased or declined suddenly through the method of citation burst. Hotspots subjects and keywords are found by utilizing the method of citation burst to probe the subject categories and keywords

1) The co-occurrence analysis of keywords:

Rendering the co-occurrence patterns of keywords by choosing the threshold interpolation and (c, cc, ccv) are set to $(2,2,20),(3,3,20),(4,4,20) .2506$ nodes and 5011 connection lines of the co-occurrence patterns are generated by the method of minimum spanning tree as shown in Fig.2. The results of clustering are reasonable since the value of Q is 0.6169 (bigger than 0.3 ) and the value of $\mathrm{S}$ is 0.542 (bigger than 0.5).

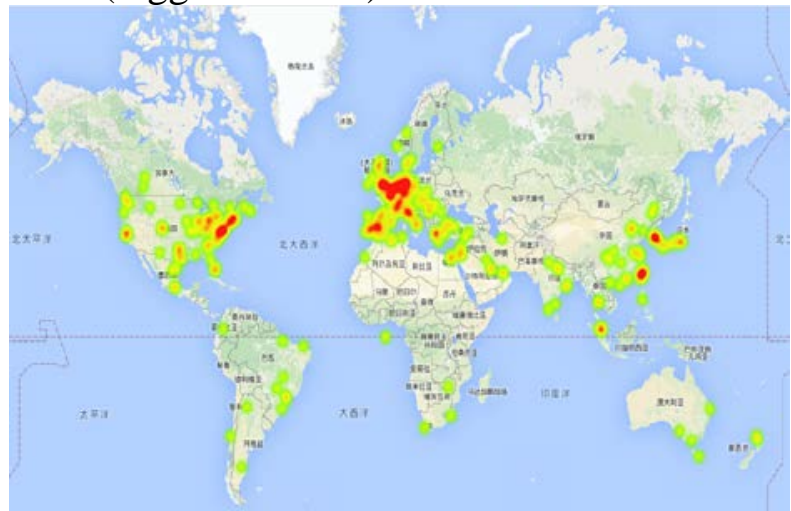

Fig. 1 Geographical distribution

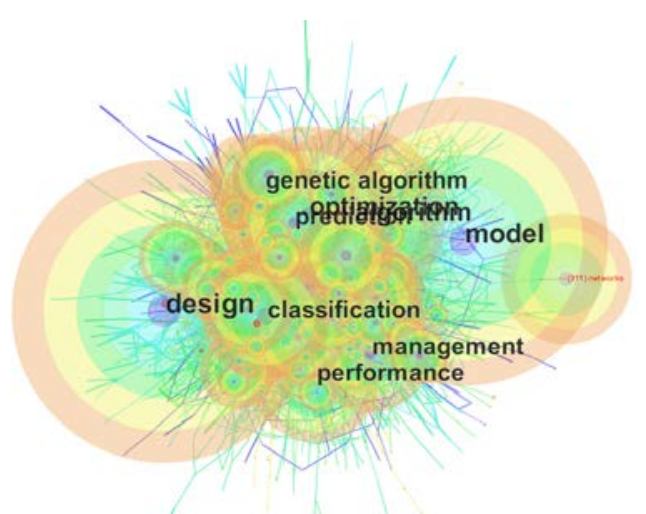

Fig. 2 Keywords co-occurrence patterns

The top 10 centrality keywords are power-systems, sliding-mode control, waves, supervisory control, traffic flow, vibration, instruction, information-systems, inference system, and human factors.

Power-systems, sliding-mode control, waves are the hotspot keywords in the field of intelligent system since they have the highest centrality among all the keywords. Top 10 centrality keywords are shown in Table 3. 
Table 3 Top 10 centrality keywords

\begin{tabular}{cccc}
\hline No. & $\begin{array}{c}\text { Centralit } \\
\mathrm{y}\end{array}$ & Frequency & Keywords \\
\hline 1 & 0.04 & 24 & power-systems \\
2 & 0.04 & 21 & sliding-mode control \\
3 & 0.04 & 16 & waves \\
4 & 0.04 & 12 & supervisory control \\
5 & 0.03 & 36 & traffic flow \\
6 & 0.03 & 34 & vibration \\
7 & 0.03 & 34 & instruction \\
8 & 0.03 & 33 & information-systems \\
9 & 0.03 & 31 & inference system \\
10 & 0.03 & 28 & human factors \\
\hline
\end{tabular}

2) The co-occurrence analysis of articles:

The timespan and the selection criteria are set to 2010-2015 (Slice Length=1) and top1000 per slice. The value of $\mathrm{N}$ in the citation co-citation map is 4109, the value of $\mathrm{E}$ is 5703 and the density is 0.0007. The result of clustering are reasonable for the value of $\mathrm{Q}$ is 0.825 (bigger than 0.3 ) and the value of $\mathrm{S}$ is 0.5192 (bigger than 0.5). The citation co-citation map is shown in Fig.3.

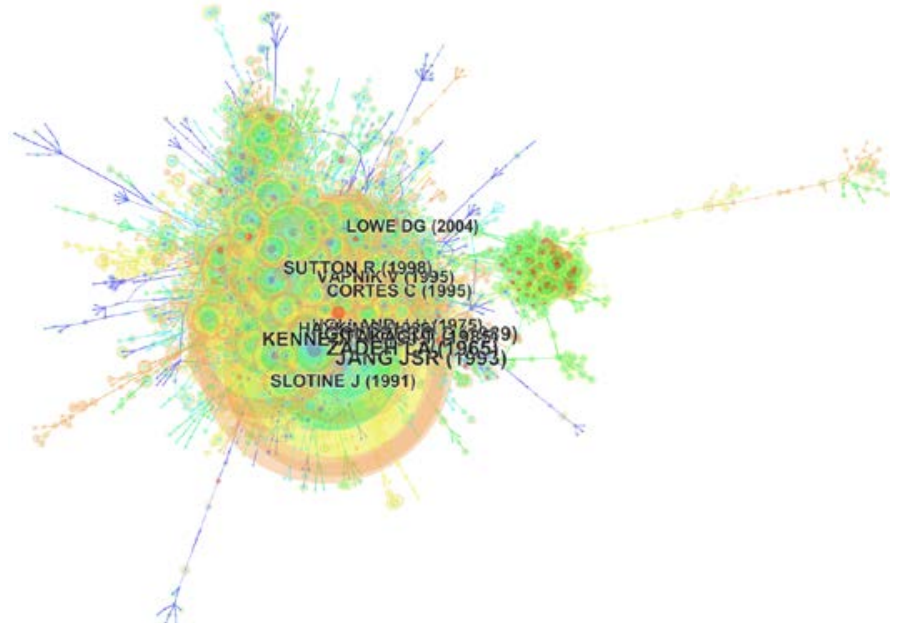

Fig. 3 Citation co-citation map

The citation burst can find out the hotspot in this field by detecting the burst of citation frequency. The co-citation analysis result shows that 161 of 4109 nodes have appeared burst. The citation burst results are sorted in descending order according to the intensity. Top 10 articles of burst from 2010 to 2015 are shown in Table 4.

Table 4 Top 10 articles of burst from 2010 to 2015

\begin{tabular}{|c|c|c|}
\hline No. & Article & Intensity \\
\hline 1 & Obstacle avoidance design for a humanoid intelligent robot with ultrasonic sensors & 5.32 \\
\hline 2 & Intelligent PID fault tolerant tracker for unknown nonlinear MIMO systems & 4.68 \\
\hline 3 & $\begin{array}{c}\text { An enhanced obstacle avoidance and path correction mechanism for an autonomous } \\
\text { intelligent robot with multiple sensors }\end{array}$ & 4.68 \\
\hline 4 & $\begin{array}{c}\text { Graph image language techniques supporting radiological, hand image } \\
\text { interpretations }\end{array}$ & 4.51 \\
\hline 5 & $\begin{array}{l}\text { Robust adaptive fuzzy tracking control with two errors of uncertain nonlinear } \\
\text { systems }\end{array}$ & 4.41 \\
\hline 6 & $\begin{array}{l}\text { Predicting electronic toll collection service adoption: An integration of the } \\
\text { technology acceptance model and the theory of planned behavior }\end{array}$ & 4.36 \\
\hline 7 & Review of an autonomous humanoid robot and its mechanical control & 4.36 \\
\hline 8 & $\begin{array}{l}\text { Integrating mobile agent technology with multi-agent systems for distributed traffic } \\
\text { detection and management systems }\end{array}$ & 4.36 \\
\hline 9 & An intelligent system for faulty-bearing detection based on vibration spectra & 4.3 \\
\hline 10 & Intelligent automatic overtaking system using vision for vehicle detection & 4.3 \\
\hline
\end{tabular}


In summary, the hotspots of intelligent system mainly concentrated in the humanoid intelligent robot $[3,4,5]$, the traffic flow management $[6,7,8]$, the vibration [9], and other topics about intelligent system $[10,11,12]$.

\section{Summary}

(1) China, the United States and Spain are in a leading position of intelligent system studies. Islamic Azad University, Chinese Academy of Sciences and Tsinghua University play a significant role in promoting the development of intelligent system studies.

(2) power-systems, sliding-mode control, waves, supervisory control, traffic flow are the hotspot keywords in the field of intelligent system since they have the highest centrality among all the keywords.

(3) From the point of the article co-citation analysis, the hotspots of intelligent system mainly concentrated in the humanoid intelligent robot, the traffic flow management, the vibration, and other topics about intelligent system.

\section{References}

[1]. Yue Chen, Ze-yuan LIU, Jin CHEN, et al. History and theory of mapping knowledge domains. Studies in Science of Science. Vol. 26 (2008) No.3, p. 449-460.

[2]. Information on: www.google.com/fusiontables.

[3]. CY Chen, BY Shih, WC Chou, et al. Obstacle avoidance design for a humanoid intelligent robot with ultrasonic sensors. Journal of Vibration \& Control. Vol. 17 (2011) No. 12, p. 1798-1804.

[4]. BY Shih, CY Chen, W Chou, et al. An enhanced obstacle avoidance and path correction mechanism for an autonomous intelligent robot with multiple sensors. Journal of Vibration \& Control. Vol. 18 (2012) No. 12, p. 1855-1864.

[5]. CY Chen, PH Huang. Review of an autonomous humanoid robot and its mechanical control. Journal of Vibration and Control. Vol. 18 (2012) No. 7, p. 973-982.

[6]. CD Chen, YW Fan, CK Farn. Predicting electronic toll collection service adoption: An integration of the technology acceptance model and the theory of planned behavior. Transportation Research Part C-emerging Technologies. Vol. 15 (2007) No. 5, p. 300-311.

[7]. B Chen, HH Cheng, J Palen. Integrating mobile agent technology with multi-agent systems for distributed traffic detection and management systems. Transportation Research Part C-emerging Technologies. Vol. 17 (2009) No. 1, p. 1-10.

[8]. V Milanes, DF Llorca, J Villagra, et al. Intelligent automatic overtaking system using vision for vehicle detection. Expert systems with applications. Vol. 39 (2012) No. 3, p. 3362-3373.

[9]. GN Marichal, M Artes, JC Garcia-Prada. An intelligent system for faulty-bearing detection based on vibration spectra. Journal of Vibration and Control. Vol. 17 (2011) No. 6, p. 931-942.

[10]. SM Guo, TJ Tsai, JSH Tsai, et al. Intelligent PID fault tolerant tracker for unknown nonlinear MIMO systems. International Journal of Nonlinear Sciences \& Numerical Simulation. Vol. 11 (2010) No. 11, p. 911-926.

[11]. Ogiela MR, Tadeusiewicz R, Ogiela L. Graph image language techniques supporting radiological, hand image interpretations. Computer Vision and Image Understanding. Vol. 103 (2006) No. 2, p. 112-120.

[12]. YF Wang, DH Wang, TY Chai, et al. Robust adaptive fuzzy tracking control with two errors of uncertain nonlinear systems. International Journal of Innovative Computing Information and Control. Vol. 6 (2010) No. 12, p. 5587-5596. 